\title{
(6) OPEN ACCESS \\ Analysis of human cytomegalovirus replication in primary cultured human corneal endothelial cells
}

\author{
Mayumi Hosogai, ${ }_{1}^{1,2}$ Nobuyuki Shima, ${ }^{3}$ Yoko Nakatani, ${ }^{1}$ Teruki Inoue, ${ }^{1,4}$ Tatsuya Iso, \\ Hideaki Yokoo, ${ }^{6}$ Hiroshi Yorifuji, ${ }^{7}$ Hideo Akiyama, ${ }^{2}$ Shoji Kishi, ${ }^{2}$ Hiroki Isomura ${ }^{1}$
}

- Additional material is published online only. To view please visit the journal online (http://dx.doi.org/10.1136/ bjophthalmol-2014-306486).

For numbered affiliations see end of article.

\section{Correspondence to} Professor Hiroki Isomura, Department of Virology and Preventive Medicine, Gunma University Graduate School

of Medicine, 3-39-22 Showa-machi, Maebashi, Gunma 371-8511, Japan; hisomura@gunma-u.ac.jp and Shoji Kishi, Department of Opthalmology, Gunma University Graduate School of Medicine;

shojikishi@gunma-u.ac.jp

Received 4 December 2014 Revised 11 June 2015 Accepted 21 July 2015 Published Online First 10 August 2015

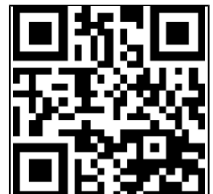

Open Access Scan to access mor free content

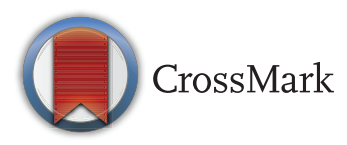

To cite: Hosogai $M$, Shima N, Nakatani $Y$, et al. Br J Ophthalmol 2015;99:1583-1590.

\section{ABSTRACT \\ Background/aims Since the first case of human cytomegalovirus (HCMV)-induced corneal endotheliitis in which HCMV DNA was detected from the patient's aqueous humour using PCR, the clinical evidence for HCMV endotheliitis has been accumulating. However, it remains to be confirmed whether HCMV can efficiently replicate in corneal endothelial cells. We, therefore, sought to determine whether primary cultured human corneal endothelial cells (HCECS) could support HCMV replication.}

Methods Human foreskin fibroblasts (HFFs) have been shown to be fully permissive for HCMV replication, and are commonly used as an in vitro model for HCMV lytic replication. Therefore, primary cultured HCECs or HFFs were infected with the vascular endotheliotropic HCMV strain TB40/E or laboratory strain Towne. We then compared viral mRNA and protein expression, genome replication and growth between the TB40/E-infected and Towne-infected HCECs and HFFs.

Results When HCECs were infected with TB40/E or Towne, rounded cells resembling owl's eyes as well as viral antigens were detected. Viral mRNA synthesis and protein expression proceeded efficiently in the HCECs and HFFs infected with TB40/E or Towne at a high multiplicity of infection (MOI). Similarly, the viral genome was also effectively replicated, with UL44-a viral DNA polymerase processivity factor-foci observed in the nuclei of HCECS. HCECs produced a substantial number of infectious virions after infection with TB40/E at both a high and low MOI.

Conclusions Primary cultured HCECs could efficiently support HCMV replication after infection at both a high and low MOl.

\section{INTRODUCTION}

Since Koizumi et $a l^{1}$ reported the first case of human cytomegalovirus (HCMV)-induced corneal endotheliitis in 2006, the clinical evidence for HCMV corneal endotheliitis in 'immunocompetent' patients has continued to accumulate. In recent years, diagnostic criteria for HCMV corneal endotheliitis based on the detection of HCMV DNA from the patient's aqueous humour using PCR in combination with clinical manifestations have been proposed. ${ }^{2}$ Nonetheless, it remains to be confirmed whether HCMV plays a role in the pathogenesis of corneal endotheliitis.

During productive infection, HCMV genes are expressed in a temporal cascade designated as immediate early (IE), early (E) and late (L). The major IE genes, UL123/122 (IE1/IE2), play a critical role in subsequent viral gene expression and viral replication efficiency. ${ }^{3}$ The $\mathrm{E}$ genes encode proteins, including viral DNA polymerase processivity factor UL44, necessary for viral DNA replication. Delayed early and L genes, which encode structural proteins in the virion, are expressed following viral DNA replication. ${ }^{4}$

HCMV replicates productively in a variety of cells, including, but not limited to, human foreskin fibroblasts (HFFs), ${ }^{5}$ human umbilical vein $^{6}$ and arterial endothelial cells, ${ }^{7}$ retinal pigment epithelial cells ${ }^{8}$ and monocyte-derived macrophages and dendritic cells. ${ }^{9}{ }^{10}$ However, it remains to be confirmed whether HCMV can replicate in human corneal endothelial cells (HCECs) in vitro.

Recently, several approaches have been developed for the cultivation of HCECs, and we have also reported culture conditions for HCECs that allow both cell adhesion and proliferation. ${ }^{11}$ Therefore, in this study, we sought to determine whether primary cultured HCECs could support HCMV replication, and herein show for the first time that HCMV can efficiently replicate in HCECs.

\section{MATERIALS AND METHODS}

\section{Cells and viruses}

Primary cultured HFFs were propagated and maintained as described previously. ${ }^{12}$ HCECs were isolated from corneas donated for research purposes (SightLife, Seattle, Washington, USA) and subjected to primary culture as described previously. ${ }^{11}$ In brief, HCECs (together with the Descemet's membrane) were stripped off and then digested at $37^{\circ} \mathrm{C}$ for $2 \mathrm{~h}$ in a basal medium containing $2 \mathrm{mg} / \mathrm{mL}$ collagenase A. Next, the cells were washed by centrifugation, incubated with $0.05 \%$ trypsin/EDTA for 5 min at $37^{\circ} \mathrm{C}$, washed and cultured with a basal medium containing basic fibroblast growth factor $(2 \mathrm{ng} / \mathrm{mL})$ in the presence of L-ascorbic acid 2-phosphate $(0.3 \mathrm{mM})$ on atelocollagen-coated dishes. The cells were used for experiments at passage 2-4. Human umbilical vein endothelial cells (HUVECs) purchased from Cell Systems (Kirkland, Washington, USA) were cultured with CS-C Complete Medium Kit R (Cell Systems) on collagen-coated dishes.

We used a vascular endotheliotropic HCMV strain, $\mathrm{TB} 40 / \mathrm{E},{ }^{13}$ and a laboratory strain, Towne with the green fluorescent protein (GFP) gene, for infection. ${ }^{3}$ To obtain TB40/E, the culture fluid from HFFs transfected with the TB40-BAC4 clone (kindly provided by Dr Barbara Adler, Max von Pettenkofer Institut, München, Germany) was collected at 7 days after observation of a $100 \%$ cytopathic effect (CPE) and then propagated as described previously. ${ }^{3}$ To observe the CPEs, the infected cells (on coverslips in 24-well plates) were 
fixed and stained with H\&E. To test the cell viability of the infected cells, trypan blue staining was performed.

\section{RNA extraction and real-time RT-PCR}

HFFs or HCECs were infected with TB40/E or Towne at a multiplicity of infection (MOI) of 3 , and RNAs were then harvested at 1, 2 and 3 days post infection (dpi) and subjected to real-time RT-PCR analysis as the replication cycle of HCMV requires $48-72 \mathrm{~h}$ to reach the final stages of maturation and release of progeny. ${ }^{14}$ Total RNA extraction, cDNA synthesis and real-time RT-PCR analysis were performed as described previously. $^{12}$ The sequences of the primer sets are shown in online supplementary table S1.

\section{Immunoblot analysis}

HFFs or HCECs were infected with TB40/E or Towne at an MOI of 3. Cells were harvested at 1, 2 and 3 dpi as approximately 6, 24 and $72 \mathrm{~h}$ are required after infection for the detection of IE, E and $\mathrm{L}$ protein expression by immunoblot or immunofluorescence analysis, respectively. ${ }^{15}$ The prepared cell lysates were electrophoretically separated, transferred to polyvinylidene difluoride membranes and reacted overnight at $4{ }^{\circ} \mathrm{C}$ with the following antibodies: anti-HCMV IE1 and IE2 (IE1/2, 1:500), early UL44 (1:500), delayed early pp65 (CA003, 1:10 000; EastCoast Bio, North Berwick, Maine, USA), late UL99 (pp28, 1:1000) and anticellular glyceraldehyde 3-phosphate dehydrogenase (GAPDH) (1:100 000). The anti-IE1/2, UL44, UL99 and GAPDH antibodies were purchased as described previously. ${ }^{16}$ After reaction with a viral antibody, the membrane was probed with horseradish peroxidase-conjugated secondary antibodies (Dako, Japan, Tokyo, Japan) for $1 \mathrm{~h}$ at room temperature $(\mathrm{R} / \mathrm{T})$ as described previously. ${ }^{12}$

\section{Immunofluorescence analysis}

Confluent cells seeded on coverslips in 24-well plates were infected with TB40/E or Towne at the indicated MOI and fixed at 1,2 and 3 dpi UL44 foci were detected as described previously. ${ }^{16}$ To estimate the development of replication compartments (RCs) in the HCECs or HFFs, at least 100 cells were scored as described previously. ${ }^{17}$

For the detection of IE1/2 and UL99 antigens, cells were fixed, treated and incubated with a blocking buffer as described previously. ${ }^{12}$ After blocking, cells were immunolabelled with anti-IE1/2 or anti-UL99 antibody $(1: 200)$ for $2 \mathrm{~h}$ at R/T and stained with the secondary antibody conjugated with Alexa Fluor 594 (Molecular Probes, Invitrogen Life Technologies, Carlsbad, California, USA) for $1 \mathrm{~h}$ at R/T. Images were obtained using an Olympus FV1000 microscope (Olympus, Tokyo, Japan).

\section{Viral DNA replication assays}

HFFs or HCECs were infected with TB40/E or Towne at an MOI of 3, and the cells were harvested at 1, 2 and 3 dpi. To prepare cell lysates, cells were suspended in a PCR lysis buffer containing $50 \mu \mathrm{g} / \mathrm{mL}$ proteinase $\mathrm{K}$ and, following the inactivation of the proteinase $K$, the input and replicated viral DNAs were detected by real-time PCR as described previously. ${ }^{4}$

\section{Electron microscopy}

The infected cells were fixed with a fixative containing $2.5 \%$ glutaraldehyde in $0.1 \mathrm{M}$ sodium cacodylate buffer $(\mathrm{pH} 7.4)$. After washing with $10 \%$ sucrose in the same buffer, the cells were postfixed with $1 \% \mathrm{OsO}_{4}$ in the same buffer and blockstained with $1 \%$ aqueous uranyl acetate. Cells were then dehydrated and embedded in Epon 812 (TAAB Laboratories Equipment, Berkshire, UK). Ultrathin sections (60-80 nm) were cut with a diamond knife (Diatome, Switzerland) on an ultramicrotome (Ultracut N Reichert-Nissei, Tokyo, Japan). The sections were double stained with $4 \%$ uranyl acetate and $0.4 \%$ lead citrate for examination by transmission electron microscope (model JEM-1010; JEOL, Tokyo, Japan).

\section{Viral growth assays}

Virus titres were determined by $50 \%$ tissue culture infectious dose $\left(\mathrm{TCID}_{50}\right)$ assay on HFFs using the formation of viral plaques on monolayers of HFFs infected with serially diluted supernatants in quadruplicate infections. ${ }^{18}$ The TCID $_{50}$ values were calculated using the method of Reed and Muench.

\section{RESULTS}

\section{Infection of HUVECs with TB40/E}

It is known that while TB40/E can replicate in HUVECs, Towne has lost this ability. ${ }^{13}$ Therefore, to confirm whether the virus obtained from the HFFs transfected with the TB40-BAC4 clone retains HUVEC tropism, HFFs or HUVECs were infected with TB40/E or Towne at an MOI of 0.5 and stained with H\&E or immunolabelled with an anti-IE1/2 antibody.

As shown in figure $1 \mathrm{~A}, \mathrm{~B}$, cell rounding and IE1/2 antigens were detected in the TB40/E-infected HUVECs. Further, a CPE and IE1/2 antigen expression were detected in HFFs, but not in the Towne-infected HUVECs. These results suggested that TB40/E maintains its original phenotype.

\section{Synthesis of viral gene transcripts in HCECs infected with $\mathrm{HCMV}$ at a high MOI}

To determine whether HCECs support HCMV mRNA expression, we compared viral gene transcription in HCECs with that in HFFs after TB40/E or Towne infection at an MOI of 3. There was no more than a twofold difference in the expression levels of IE1 and IE2, early gB and UL44, and late UL75 and UL99 mRNAs between the TB40/E-infected HCECs and HFFs (see online supplementary figure S1A-F). Similarly, the expression levels of IE1, IE2, gB, UL44, UL75 and UL99 mRNAs in the Towne-infected HFFs and HCECs were also comparable (see online supplementary figure S1G-L). From these results, we concluded that viral IE, E and L mRNAs were efficiently expressed after the infection of HCECs with TB40/E or Towne at a high MOI, and TB40/E and Towne did not show the expected differences in that the susceptibility of HCECs appeared to be independent of the features dictating infection in HUVECs.

\section{Synthesis of viral proteins in HCECs infected with HCMV at a high $\mathrm{MOI}$}

To determine whether HCECs support HCMV protein expression, we first infected HCECs with TB40/E or Towne at an MOI of 0.5 and then stained them with H\&E or immunolabelled them with an anti-IE1/2, early UL44 and late UL99 antibody.

As shown in figure $1 \mathrm{C}$, rounded cells resembling owl's eyes were observed up to $3 \mathrm{dpi}$, and IE1/2, UL44 and UL99 antigens began to be detected at 1,1 and $3 \mathrm{dpi}$, respectively. There were smaller numbers of UL99 antigen-positive cells in the TB40/ E-infected HCECs than in the Towne-infected HCECs (figure $1 \mathrm{C}$ ), presumably due to the lower protein expression level (see figure 2A). Trypan blue staining did not reveal any loss of cell viability up to $3 \mathrm{dpi}$ (figure $1 \mathrm{D}$ ). 
A

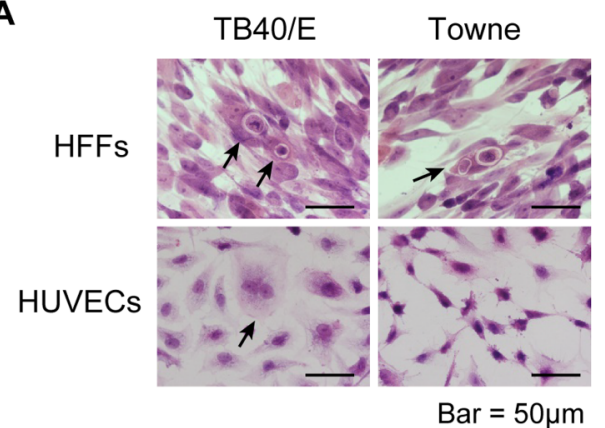

C

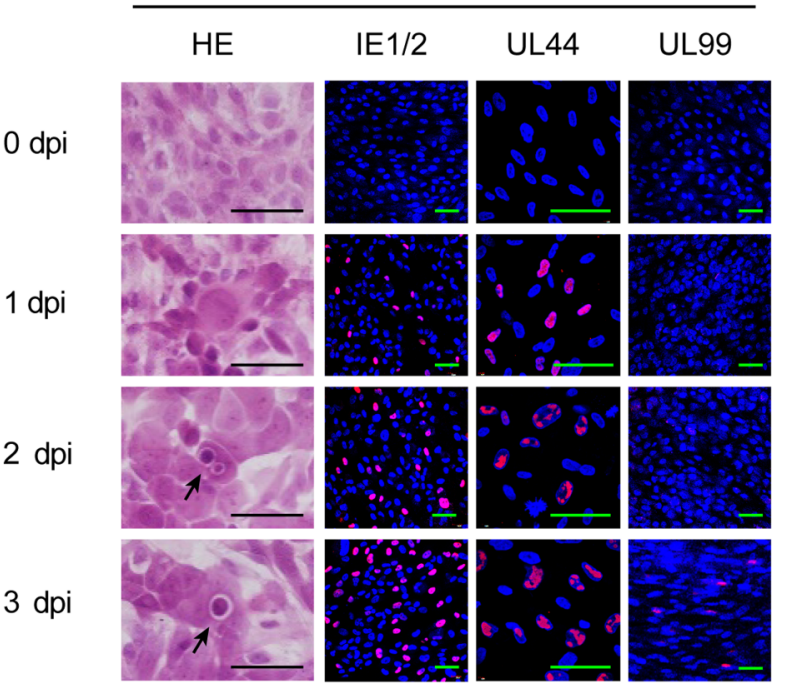

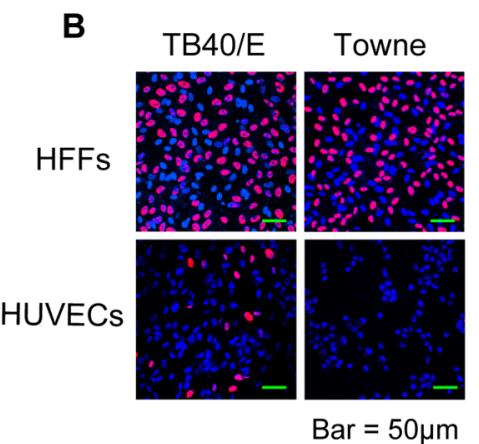

Towne

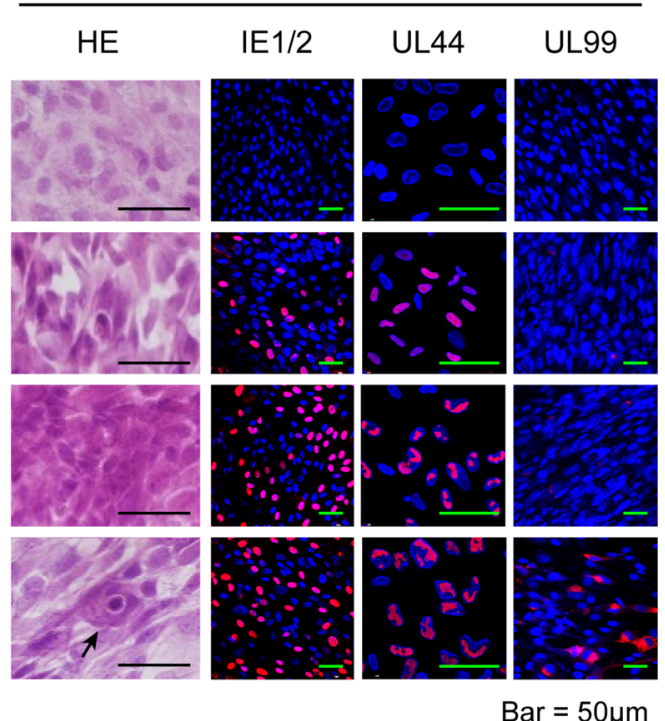

D

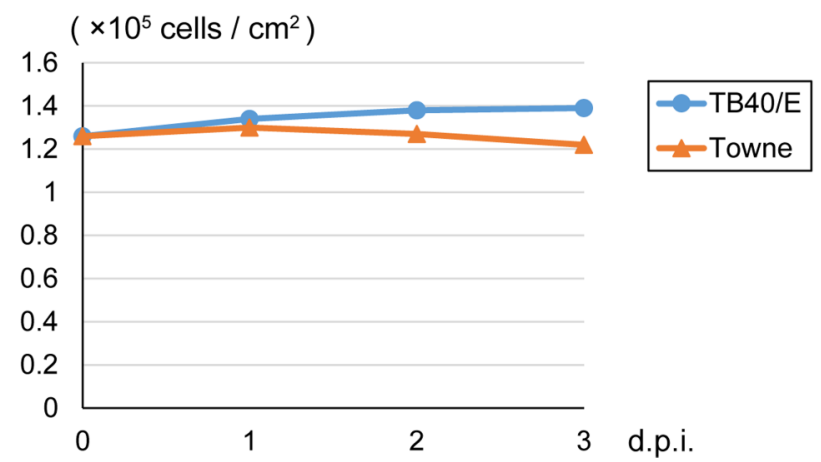

Figure 1 Primary cultured human foreskin fibroblasts (HFFs), human umbilical vein endothelial cells (HUVECs) and human corneal endothelial cells (HCECs) inoculated with human cytomegalovirus strain TB40/E or Towne. HFFs, HUVECs and HCECs were infected with TB40/E or Towne at a multiplicity of infection of 0.5. (A) To observe the cytopathic effects, the TB40/E-infected or Towne-infected HFFs and HUVECs were fixed and stained with H\&E at 3 days post infection (dpi). (B) To confirm the infectivity of TB40/E and Towne, the infected cells were immunolabelled with an anti-IE1/2 antibody at $1 \mathrm{dpi}$. (C) The TB40/E-infected or Towne-infected HCECs were fixed before infection and at 1, 2 and 3 dpi and immunolabelled with an anti-IE1/2, UL44 or UL99 (pp28) antibody. (A and C) Arrows show rounded cells resembling owl's eyes. (B and C) Nuclei were stained with 4',6-diamidino-2-phenylindole (blue signals), and the cells immunolabelled with a viral antibody were stained with an antimouse IgG secondary antibody conjugated with Alexa Fluor 594 (red signals). (D) Cell viability of the TB40/E-infected or Towne-infected HCECs was tested by trypan blue staining before infection and at 1, 2 and $3 \mathrm{dpi}$. IE, immediate early.

We next infected HFFs or HCECs with TB40/E or Towne at an MOI of 3, and then undertook an analysis by western blotting with antibodies against viral IE1/2, early UL44, delayed early pp65 and late UL99 and cellular GAPDH.

In the TB40/E-infected or Towne-infected cells, IE1, IE2, UL44 and pp65 proteins were expressed to a similar extent in
HFFs and HCECs at 1, 2 and 3 dpi (figure 2A, B). Similarly, there was no significant difference in the expression level of UL99 protein between the Towne-infected HFFs and HCECs (figure 2B). In contrast, after infection with TB40/E, the amount of UL99 protein in HCECs was a little lower than that in HFFs at 3 dpi (figure 2A), which is in agreement with 
Figure 2 Analysis of viral immediate early (IE), early and late gene translation after human cytomegalovirus infection. Human foreskin fibroblasts (HFFs) or human corneal endothelial cells (HCECS) were infected with TB40/E (A) or Towne (B) at a high multiplicity of infection, and analysed for viral proteins. IE1/2, early UL44, delayed early pp65 and late UL99 (pp28) proteins at 1, 2 and 3 days post infection (dpi) as described in the Materials and methods. GAPDH served as an equal protein loading. Uncropped images of blots are shown in online supplementary figure S2.
A TB40/E

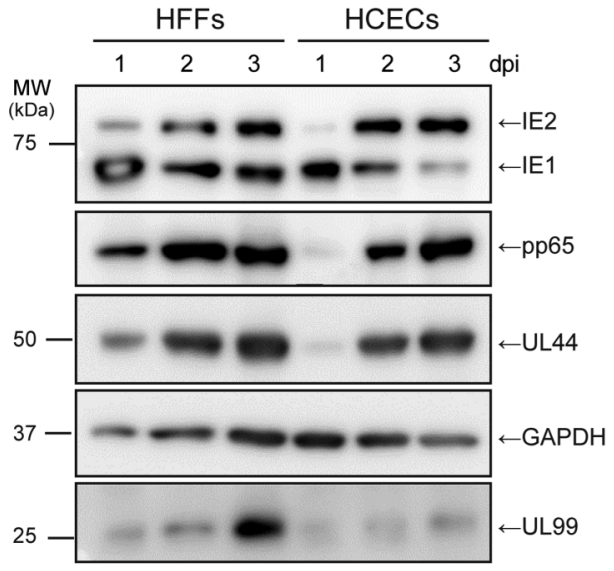

B Towne

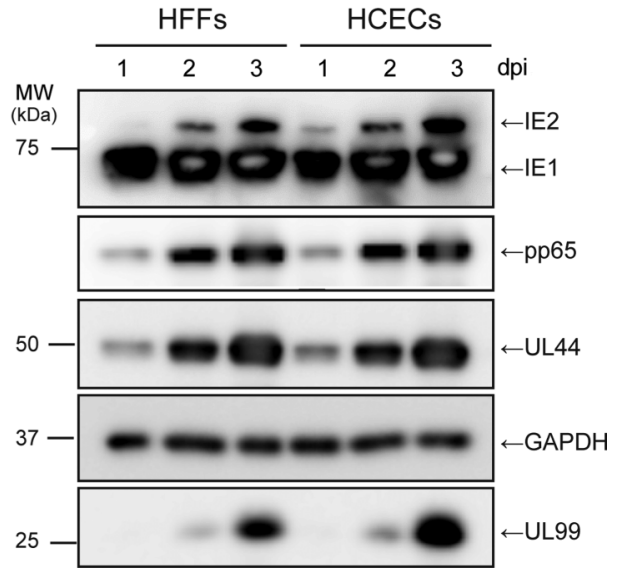

the results for viral mRNA levels (see online supplementary figure S1F).

The ratio of IE1 to IE2 protein in the Towne-infected cells was higher than that in the TB40/E-infected cells (figure 2A, B). IE1 and IE2 mRNAs are alternatively spliced transcripts that share their first three exons (exon 1, 2 and 3) and differ in their $3^{\prime}$ terminal exon. IE1 and IE2 mRNA have exon 4 and 5 in the $3^{\prime}$ end, respectively. ${ }^{19}$ The lower expression ratio of IE2 protein in Towne-infected cells might be partially due to the difference in the HCMV-strain dependent ratio of inclusion-to-skipping of the exon 4. Based on these results, we concluded that viral IE, E and L proteins were efficiently
A

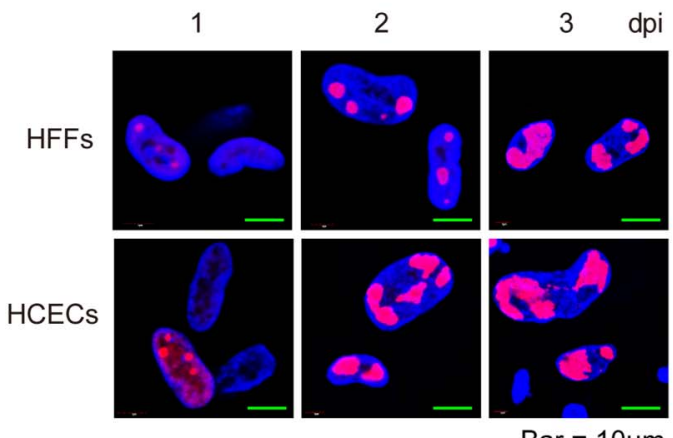

Bar $=10 \mu \mathrm{m}$
B

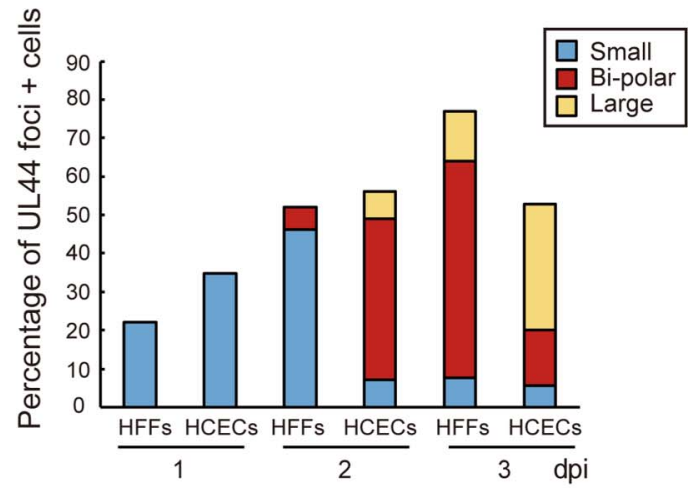

C

TB40/E

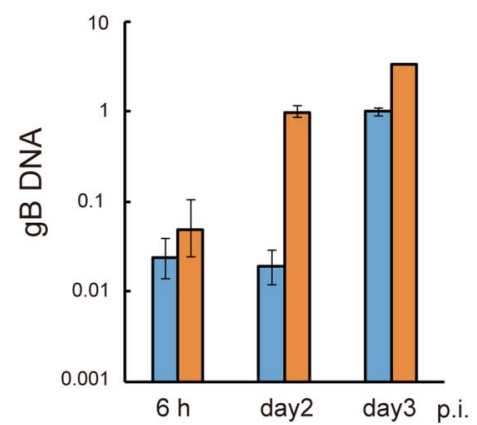

Towne

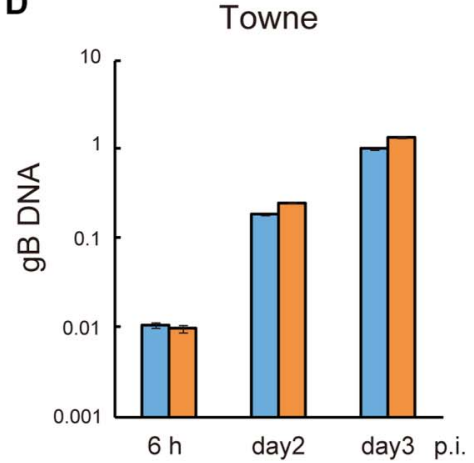

Figure 3 Proper localisation of viral UL44 antigen for effective viral DNA replication after human cytomegalovirus infection. (A and B) Human foreskin fibroblasts (HFFs) and human corneal endothelial cells (HCECs) were infected with TB40/E at a high multiplicity of infection (MOI). (A) HCECs displayed proper localisation of viral UL44 antigen at 1, 2 and 3 days post infection (dpi). Nuclei were stained with 4',6-diamidino-2phenylindole (blue signals) and the cells immunolabelled with a UL44 antibody were stained with an antimouse IgG secondary antibody conjugated with Alexa Fluor 594 (red signals). (B) Graphical representation of the results of scoring for the presence and size of UL44 foci in HFFs and HCECs. The blue, red and yellow bars represent the percentages of cells with multiple small UL44 foci, bipolar foci and one large focus at the given time points, respectively. (C and D) Analysis of viral DNA replication after the infection of HFFs or HCECs with TB40/E (C) or Towne (D) at a high MOI. Viral DNA was quantified by real-time PCR using gB primers and probe. Real-time PCR with beta-globin was also performed to serve as an internal control. The sequences of the primer sets are shown in online supplementary table S1. Data are the averages of three independent DNA samples from the infected cells. 
expressed in HCECs after infection with TB40/E or Towne at a high MOI.

\section{HCECs supported effective viral DNA replication after infection with HCMV at a high MOI}

HCMV DNA replication proteins, including UL44, are localised in large nuclear structures that resemble HSV-1 RCs. ${ }^{20}$ To determine whether all stages of the compartments, from small multiple foci (which represent prereplication sites) to large single foci (which represent fully developed sites), could be observed in TB40/E-infected HCECs and thereby establish the presence of active viral genome replication, ${ }^{21} \mathrm{HFF}$ or HCECs were infected with TB40/E at an MOI of 3 and stained with anti-UL44 antibody.

Small foci were detected at $1 \mathrm{dpi}$, and bipolar and large single foci were detected at 2 and 3 dpi, respectively, in
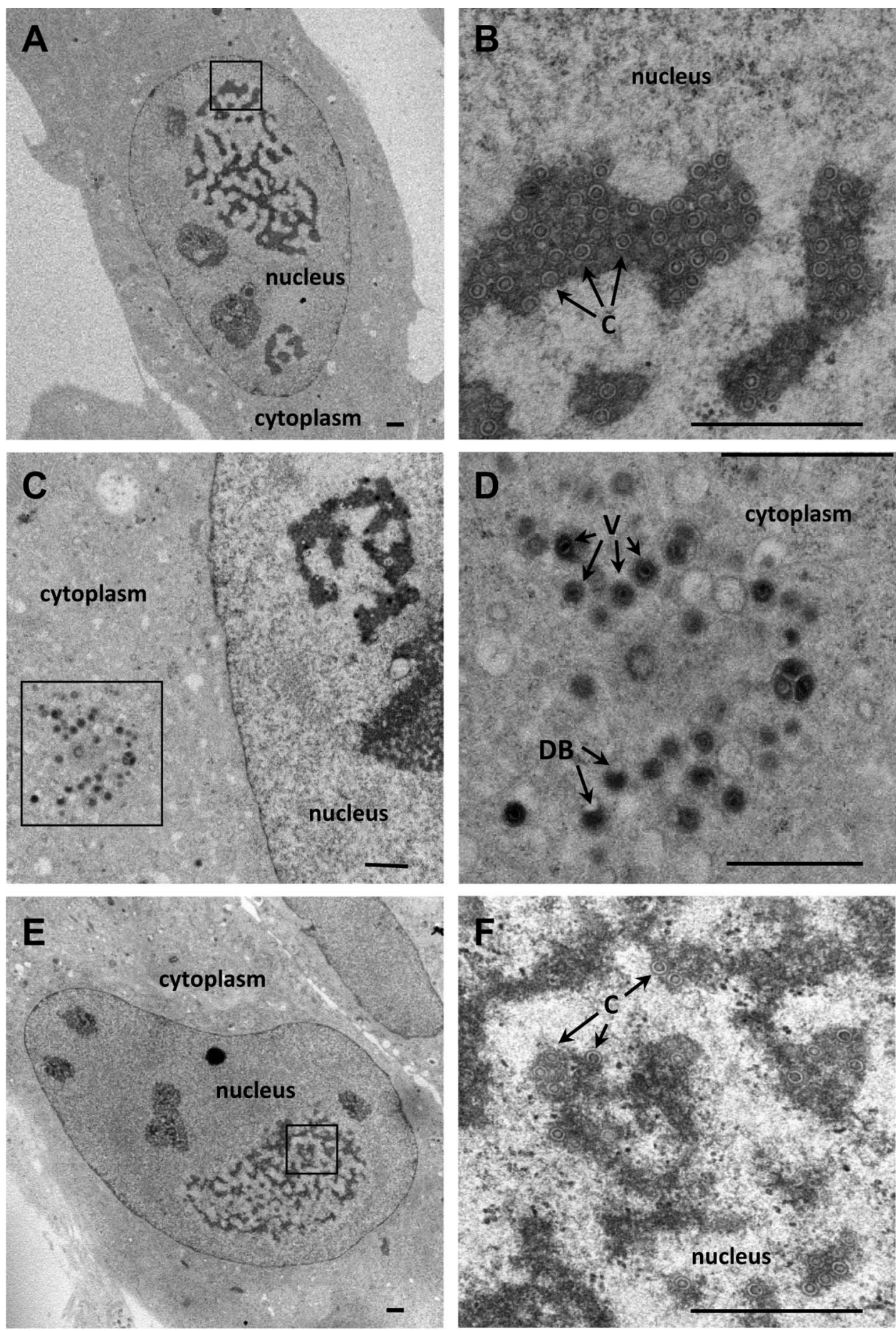

Bar $=1 \mu \mathrm{m}$

Figure 4 Human corneal endothelial cells (HCECs) produced a substantial number of infectious virions after human cytomegalovirus infection. At 3 days post infection (dpi) with TB40/E at a high multiplicity of infection, larger numbers of capsids preparing to package ${ }^{22}$ were observed in the HCECs (A and B) than in the HFFs (E and F). Cytoplasmic virus particles were observed at 3 dpi in the TB40/E-infected HCECs (C and D). Viral capsids preparing to package (C), cytoplasmic viral particles $(\mathrm{V})$ and dense bodies (DB) are shown by arrows. 
A

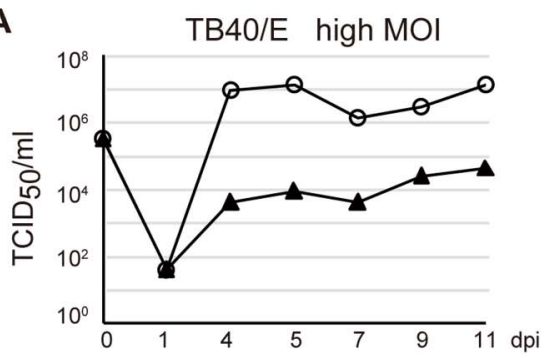

C

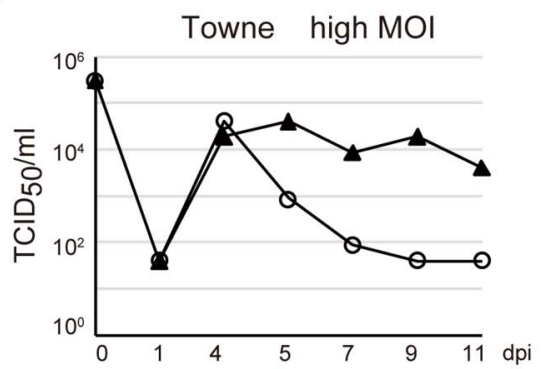

B

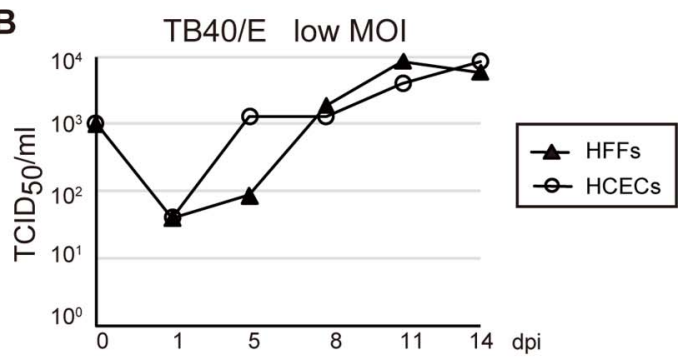

D

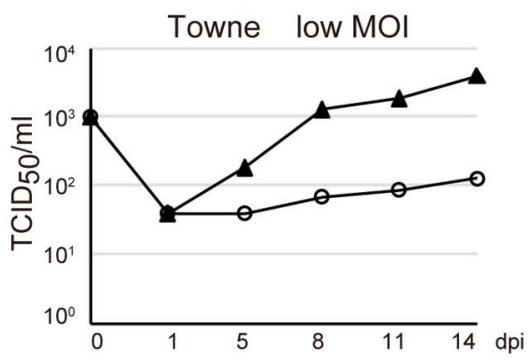

$\mathbf{E}$

Towne high $\mathrm{MOI}$

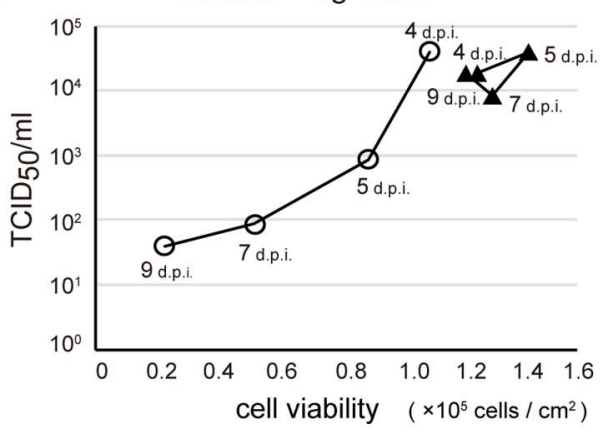

$\mathbf{F}$

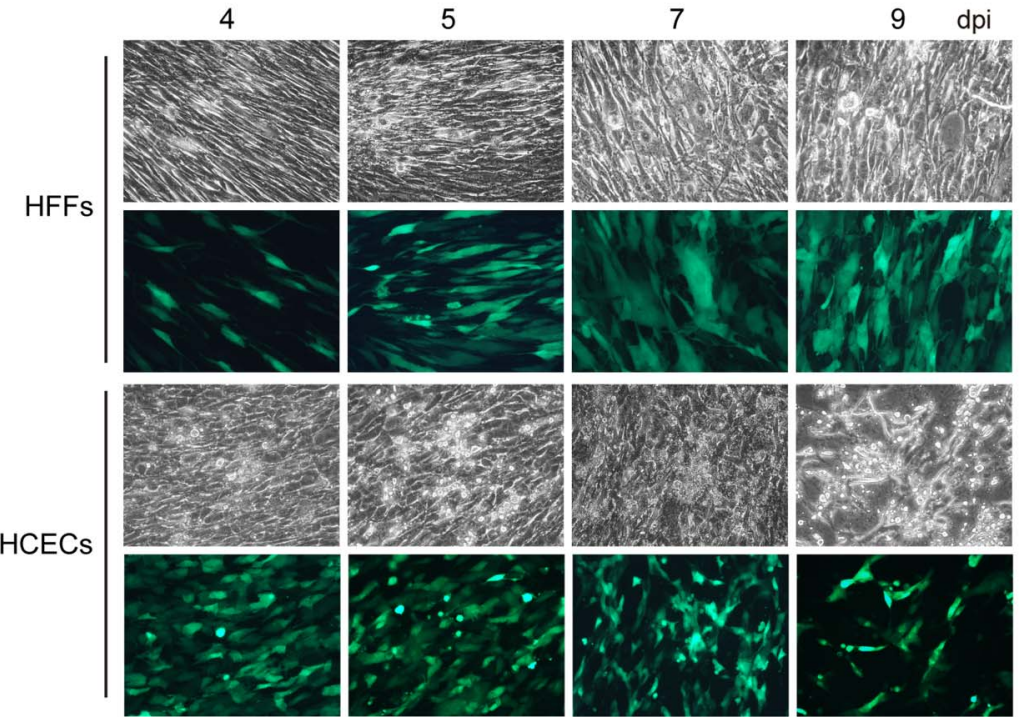

Figure 5 Growth of TB40/E or Towne after the infection of human foreskin fibroblasts (HFFs) or human corneal endothelial cells (HCECs). HFFs or HCECs were infected with TB40/E ( $A$ and $B$ ) or Towne ( $C$ and $D)$ at a high ( $A$ and $C$ ) or low (B and D) multiplicity of infection (MOI). Virus titres were determined by tissue culture infectious dose $\left(\mathrm{TCI}_{50}\right)$ assay as described in Materials and methods, and similar findings were obtained for repeated experiments. (E and F) The lower virus titres corresponded to the loss of cell viability in the Towne-infected HCECS. (E) The cell viability of the Towne-infected HFFs or HCECs at 4, 5, 7 and 9 days post infection (dpi) was tested using trypan blue staining. (F) green fluorescent protein-positive HFFs or HCECs in which Towne was possibly replicated are shown at 4, 5, 7 and 9 dpi. 
TB40E-infected HCECs. However, there was a lag in the development of these RCs in HFFs infected with TB40/E in comparison with those observed in HCECs (figure 3A, B).

To determine whether the TB40/E or Towne genome efficiently replicates in HCECs, HCECs or HFFs were infected with TB40/E or Towne at an MOI of 3 and subjected to viral DNA replication assays.

The amount of viral DNA was increased approximately 100 -fold at 2 dpi in HCECs after TB40/E infection, suggesting that the viral DNA derived from HCECs at 2 dpi had replicated. In contrast, the amount of viral DNA was not increased at 2 dpi, but was increased at $3 \mathrm{dpi}$ in TB40/E-infected HFFs (figure 3C). Since UL44 is necessary for viral DNA replication, these findings could be related to the delay in the replication of the TB40/E genome in HFFs. In both Towne-infected HFFs and HCECs, there was an increase in the amount of viral DNA at 2 dpi (figure 3D). Taken together, these findings led us to conclude that both the TB40/E and Towne genomes efficiently replicated in the HCECs.

\section{HCECs produced substantial numbers of infectious virions after HCMV infection at a high and low MOI}

To investigate the presence of the intracellular trafficking of virus particles, sections of TB40/E-infected HCECs (figure 4AD) or HFFs (figure 4E, F) were analysed under an electron microscope. RCs were observed in the nucleus, and, compared with those observed in the HFFs, larger numbers of capsids preparing to package ${ }^{22}$ were identified in the lobar regions of the RCs in the HCECs at 3 dpi (figure 4B, F). Cytoplasmic viral particles and electron-dense intracytoplasmic particles (dense bodies) were also clearly visible at $3 \mathrm{dpi}$ in TB40/E-infected HCECs (figure 4C, D).

To, therefore, compare the growth of TB40/E or Towne at a high and low MOI in HCECs with that in HFFs, HCECs or HFFs were variously infected with TB40/E or Towne at an MOI of 3 or 0.01 , and viruses from the infected cultures were titrated at the indicated times.

The growth of TB40/E was delayed in HFFs compared with that in HCECs after infection at both a high and low MOI (figure 5A, B). We detected an approximately 100-fold to 1000 -fold higher level of viral replication in HCECs compared with that in HFFs at a high MOI (figure 5A). In contrast, the viral titre of TB40/E in HFFs was only approximately 10-fold lower than that in HCECs after 5 dpi at a low MOI (figure 5B). In HCECs infected with Towne at a high MOI, the viral titre dropped dramatically by more than one order of magnitude at 5 dpi (figure 5C). This corresponded to the loss of cell viability at 4, 5, 7 and 9 dpi (figure 5E, F). However, GFP-positive cells in which Towne possibly replicated remained even at 9 dpi in the Towne-infected HCECs (figure 5F). Towne, which has already adapted to efficient replication in HFFs, could undergo abortive infection in HCECs from 5 dpi onwards at a high MOI. The viral titre of Towne-infected HCECs at a low MOI was approximately 10-fold lower than that in HFFs throughout the course of infection (figure 5D). Based on these results, we concluded that TB40/E, but not Towne, grew more efficiently in HCECs than in HFFs at both a high and low MOI.

\section{DISCUSSION}

Owl's eye morphological features, defined as large HCECs in which the nuclei have an area of high reflection surrounded by a halo of low reflection, were observed by in vivo laser confocal microscopy in patients with HCMV corneal endotheliitis. ${ }^{23}$ Since 'owl's eye cells' are typically seen in specimens from kidneys, lungs and other organs of patients with congenital or acquired HCMV infection, ${ }^{24}$ the owl's eye morphological features were hypothesised to represent HCMV-infected HCECs. ${ }^{23}$ Nonetheless, we have no direct evidence that HCMV efficiently replicates in the HCECs of patients as diagnostic corneal endothelial biopsy is invasive and not commonly performed in patients with corneal endotheliitis. We, therefore, undertook in vitro studies using primary cultured HCECs and found 'owl's eye cells' in the infected HCECs.

Towne, unlike TB40/E, does not replicate well in cultured HUVECs. Most of the genes located between UL128 and UL150 are either missing or mutated in Towne after extensive serial propagation on fibroblasts. In contrast, TB40/E, which has not been extensively propagated on fibroblasts, harbours fewer mutations, and retains the ability to replicate in a wider variety of cultured cell types. ${ }^{13}$ Our current data indicated that Towne, as well as TB40/E, could replicate in HCECs up to 4 dpi at a high MOI (figure 5A, C), suggesting that there is a difference in the susceptibility to Towne between HUVECs and HCECs. HCMV has been shown to be highly polymorphic both among and within human hosts; ${ }^{25}$ therefore, further study is needed to determine the genomic populations of the virus that actually replicate in the aqueous humour of patients with HCMV corneal endotheliitis.

To the best of our knowledge, this is the first report to show that primary cultured HCECs efficiently support HCMV replication. It is possible that HCMV corneal endotheliitis is associated with HCMV replication in the HCECs followed by the release of replicated virions into the aqueous humour of the patients.

\section{Author affiliations}

${ }^{1}$ Department of Virology and Preventive Medicine, Gunma University Graduate School of Medicine, Maebashi, Gunma, Japan

${ }^{2}$ Department of Ophthalmology, Gunma University Graduate School of Medicine, Maebashi, Gunma, Japan

${ }^{3}$ Department of Ophthalmology, University of Tokyo Hospital, Tokyo, Japan

${ }^{4}$ Department of Medicine and Molecular Science, Gunma University Graduate School of Medicine, Maebashi, Gunma, Japan

${ }^{5}$ Department of Medicine and Biological Science, Gunma University Graduate School of Medicine, Maebashi, Gunma, Japan

${ }^{6}$ Department of Human Pathology, Gunma University Graduate School of Medicine, Maebashi, Gunma, Japan

${ }^{7}$ Department of Anatomy, Gunma University Graduate School of Medicine, Maebashi, Gunma, Japan

Acknowledgements We thank Koji Isoda for his technical advice on H\&E staining.

Contributors Conceived and designed the experiments: $\mathrm{MH}, \mathrm{HI}$. Performed the experiments: MH, YN, TIno, HYori. Analysed the data: MH, HYori, HI. Contributed reagents/materials/analysis tools: NS, TIso, HYoko, HYori, HA, SK. Wrote the paper: $\mathrm{MH}, \mathrm{HI}$.

Funding This work was supported by a Grant-in-Aid for Challenging Exploratory Research ( 2013) (24659309 to HI), and a Grant-in-Aid for Scientific Research (26462678 to HA)

\section{Competing interests None.}

Provenance and peer review Not commissioned; externally peer reviewed.

Open Access This is an Open Access article distributed in accordance with the Creative Commons Attribution Non Commercial (CC BY-NC 4.0) license, which permits others to distribute, remix, adapt, build upon this work non-commercially, and license their derivative works on different terms, provided the original work is properly cited and the use is non-commercial. See: http://creativecommons.org/ licenses/by-nc/4.0/

\section{REFERENCES}

1 Koizumi N, Yamasaki K, Kawasaki S, et al. Cytomegalovirus in aqueous humor from an eye with corneal endotheliitis. Am J Ophthalmol 2006;141:564-5.

2 Koizumi N, Inatomi T, Suzuki T, et al. Clinical features and management of cytomegalovirus corneal endotheliitis: analysis of 106 cases from the Japan corneal endotheliitis study. Br J Ophthalmol 2015;99:54-8. 
3 Isomura H, Tsurumi T, Stinski MF. Role of the proximal enhancer of the major immediateearly promoter in human cytomegalovirus replication. J Virol 2004;78:12788-99.

4 Isomura H, Stinski MF, Kudoh A, et al. The late promoter of the human cytomegalovirus viral DNA polymerase processivity factor has an impact on delayed early and late viral gene products but not on viral DNA synthesis. J Virol 2007:81:6197-206.

5 Stinski MF. History of the molecular biology of cytomegaloviruses. Methods Mol Biol 2014;1119:1-14

6 Percivalle $E$, Revello MG, Vago $L$, et al. Circulating endothelial giant cells permissive for human cytomegalovirus (HCMV) are detected in disseminated HCMV infections with organ involvement. J Clin Invest 1993;92:663-70.

7 Kahl M, Siegel-Axel D, Stenglein S, et al. Efficient lytic infection of human arterial endothelial cells by human cytomegalovirus strains. J Virol 2000;74:7628-35.

8 Tugizov S, Maidji E, Pereira L. Role of apical and basolateral membranes in replication of human cytomegalovirus in polarized retinal pigment epithelial cells. J Gen Virol 1996;77(Pt 1):61-74.

9 Weinshenker BG, Wilton S, Rice GPA. Phorbol ester-induced differentiation permits productive human cytomegalvirus infection in a monocytic line. Immunol 1988:140:1625-31.

10 Hertel L, Lacaille VG, Strobl H, et al. Susceptibility of immature and mature Langerhans cell-type dendritic cells to infection and immunomodulation by human cytomegalovirus. J Virol 2003;77:7563-74.

11 Shima N, Kimoto M, Yamaguchi M, et al. Increased proliferation and replicative lifespan of isolated human corneal endothelial cells with L-ascorbic acid 2-phosphate. Invest Ophthalmol Vis Sci 2011;52:8711-17.

12 Seto $E$, Inoue $T$, Nakatani $Y$, et al. Processing bodies accumulate in human cytomegalovirus-infected cells and do not affect viral replication at high multiplicity of infection. Virology 2014;458-459:151-61.

13 Sinzger $C$, Hahn G, Digel $M$, et al. Cloning and sequencing of a highly productive, endotheliotropic virus strain derived from human cytomegalovirus TB40/E. J Gen Virol 2008;89:359-68.

14 Mocarski ESST, Griffiths PD, Pass RF. Cytomegaloviruses, field's virology. 5th edn. Philadelphia: Lippincott Williams \& Wilkins, 2013.
15 Wathen MW, Stinski MF. Temporal patterns of human cytomegalovirus transcription: mapping the viral RNAs synthesized at immediate early, early, and late times after infection. J Virol 1982;41:462-77.

16 Isomura H, Stinski MF, Murata T, et al. The human cytomegalovirus gene products essential for late viral gene expression assemble into prereplication complexes before viral DNA replication. J Virol 2011;85:6629-44.

17 Penfold ME, Mocarski ES. Formation of cytomegalovirus DNA replication compartments defined by localization of viral proteins and DNA synthesis. Virology 1997;239:46-61.

18 Nevels M, Brune W, Shenk T. SUMOylation of the human cytomegalovirus 72-kilodalton IE1 protein facilitates expression of the 86-kilodalton IE2 protein and promotes viral replication. J Virol 2004;78:7803-12.

19 Stenberg RM, Witte PR, Stinski MF. Multiple spliced and unspliced transcripts from human cytomegalovirus immediate-early region 2 and evidence for a common initiation site within immediate-early region 1. J Virol 1985;56: 665-75.

20 Wilcock D, Lane DP. Localization of p53, retinoblastoma and host replication proteins at sites of viral replication in herpes-infected cells. Nature 1991;349:429-31.

21 Luo MH, Schwartz PH, Fortunato EA. Neonatal neural progenitor cells and their neuronal and glial cell derivatives are fully permissive for human cytomegalovirus infection. J Virol 2008;82:9994-10007.

22 Tandon R, Mocarski ES, Conway JF. The A, B, Cs of herpesvirus capsids. Viruses 2015;7:899-914

23 Kobayashi A, Yokogawa $H$, Higashide $T$, et al. Clinical significance of owl eye morphologic features by in vivo laser confocal microscopy in patients with cytomegalovirus corneal endotheliitis. Am J Ophthalmol 2012;153: 445-53.

24 Herriot R, Gray ES. Images in clinical medicine. Owl's-eye cells. N Engl J Med 1994;331:649.

25 Renzette N, Gibson L, Bhattacharjee B, et al. Rapid intrahost evolution of human cytomegalovirus is shaped by demography and positive selection. PLOS Genet 2013;9:e1003735. 